\title{
Commentary
}

\section{Epigenetics at work}

\author{
Rayna Rapp \\ New York University, New York, USA. \\ E-mail: rayna.rapp@nyu.edu
}

BioSocieties (2018) 13, 780-786. https://doi.org/10.1057/s41292-017-0093-9;

Published online 5 October 2018

As readers of BioSocieties have surely noted, environmental epigenetics has become our life science $d u$ jour over the last decade. It is the field that we social, cultural, and historical researchers love to configure as a site of potential reconciliation of possible life/social science collaboration, a promissory note addressing the longstanding problem of how the social gets under the skin of individuals, populations, and the thought styles of all of us who study such things. How might we use new genomic and epigenomic tools to query gene/environment interactions or-better still-co-productions? Answers are pouring forth not only from geneticists, neuropsychologists, and microbiome researchers, but from public health epidemiologists, patient groups participating in research, and the social scientists peering over their shoulders, as well. Indeed, epigenetics is quintessentially good to think with, an old-made-new science that takes on board the multiple complexities of the postgenomic scientific landscape, acknowledging if not exactly focusing on the environmental.

That very key word, "environment," has, of course, its own complex history, now fueling current political consciousness as the planet heats up. Along the way, social contributions to rapid environmental changes, including mutations and migrations in causes and expressions of health and illness, are increasingly under scrutiny. The very polysemy of "environment"-from commonsense to controversial, from technical mechanisms of gene expression and gene silencing to the interactive traces of molecular biomarkers-makes it an appealing discursive candidate for tackling seemingly intractable problems of complexity. Hence, thinking through "environmental epigenetics" makes sense to many investigators at the present moment.

How have qualitative lab-based social scientists come to navigate this field, and how have we fared in this exploration? Jorg Niewohner and Margaret Lock provide a synthetic and adroit philosophical overview of what social science in general and ethnographic investigations in particular have accomplished as they demonstrate the permanent entanglement and inherent co-production of encultured biology, or biologized culture. In "Situating local biologies: Anthropological perspectives on environment/human entanglements," the duo reviews some recent thinking about how

(O) ur permeable skin-bound selves comprise a collection of ecosystems of miniaturised communities that are products of our evolutionary past, more recent historical events, 
and of social and political contingencies of many kinds. Environmental epigenetics reopens a space for discussion and research at the intersection of biology and social science...Bodies emerge in evolutionary, social, biographical, and cellular time. Hence, process ontology, new materialisms, and ecosocial theory are important elective affinities for situating biologies.

Theirs is a particularly useful and very sophisticated introduction to the dilemmas of moving beyond static, categorical thought when approaching these multi-leveled entanglements. They offer a genealogy of classic references, and identify key conundrums. They also revisit Lock's initial concept of "local biologies," here evolving it into "situated biologies." By this they mean that organisms (human and otherwise) are always located in time, space, and rapidly globalizing social practices, hence their emergent biologies are contingent on how these continual forces are expressed at every level of life. They push us readers to move beyond a tacit "interactionism" toward a processual ontological notion of continuous becoming; epigenetics plays a part in this process of culturalized biology but it is just one field amongst many forms of expert knowledge production that are arising as researchers confront the many unknown unknowns of "these entanglements as moving targets."

The tone of Niewohner and Lock's argument is not celebratory; au contraire, they are prudent in their descriptions. As many of us who collaborate with bench scientists have discovered, rich opportunities for empirical and collaborative research across the social/life sciences are continually at risk of being mobilized in support of the reestablishment of a narrative that privileges the biological, bringing new understandings of the inherently entangled biosocial under its powerful reach. Many material forces place barriers in the way of scientific engagement with the complexities of social life as they co-produce embodied states at the individual and group level. The social facts of laboratory life are likely to overdetermine this process. The building blocks for what counts as new knowledge production in environmental epigenetics include the uber-disciplinary requisites of successful grants writing, publications, time-consuming engagement with policy and regulatory mechanisms, and sometimes, alliances with or responses to citizen mobilization. All contribute to contingencies of biosocial knowledge-making.

The materiality on which environmental epigenetic research rests is teased out in Sahra Gibbon's essay, "Calibrating cancer risk, uncertainty and environments: the contexts between genetics and epigenetics in southern Brazil." Gibbon conducted ethnographic studies of/with genetic, epigenetic, epidemiological, and clinical researchers focused on the $\mathrm{R} 337 \mathrm{~h}$ mutation in the TP53 (tumor suppressor) gene, associated with Li Fraumeni Syndrome, found at relatively high rates in Southern Brazil. Presence of this mutation confers strong lifetime risk for developing a rare cancer (or a fairly common one like breast cancer). The changed gene also seems implicated in variable age of onset, and type of rare cancer that carriers will develop. Gibbon wanted to know how scientists from a diversity of disciplines approached this situation of inherent uncertainty as they tried to figure out how/ why this variant is or is not expressed and how to design screening and treatment options for it. What place might environmental thinking play, replete with the ambiguity of what counts as "environment"? She also engaged with high-risk LFS families, some with members that had diagnosed rare cancers, and others who had screened as positive for the syndrome, but were currently unaffected. 
Throughout, Gibbon shows us epigenetic thinking at work in all its polysemic heterogeneity. Diverse modes of explanation amongst researchers, clinicians, patients express a heady mix of genetics, epigenetics, and historical and sociocultural explanations. Some researchers are narrowly but deeply focused on intracellular processes that reflect molecular environments, attempting to understand how risk translates into disease at different ages of onset and diverse tissue types. Others see European migration ancestry and founder effects as key to understanding the elevated rates and diverse expressions of $\mathrm{Li}$ Fraumeni-associated cancers; some connect its expression to the "cultural finger" of dietary and other lifestyle patterns passed down through the generations, but "not in the DNA," as some interviewed researchers and advocates put it. Clinicians expressed hesitancy about using the Li Fraumeni mutation diagnostically since its implications for treatment and prognosis are so little understood. But patients were quick to link their cancers/risks to frankly environmental factors like diet, pollution, poverty, and stress, especially stressful negative family relations. Gibbon sees this polysemy of cause-effect slippage and elaboration as linked to a specific Brazilian history not only of ancestry migration toward the south of the country, but also to an influential Lamarckian public health tradition in earlier twentieth century styles of medical thought and practice. Almost all the people she interviewed, ranging from diverse professionals to patients and advocates, were "quick to fill the gap" (as she puts it) between the still-mysterious presence of the mutation and its diverse expressivity by recourse to environmental explanations.

Gibbon's analysis is particularly sensitive to the longstanding dependence of Brazilian genetic/and now epigenetic research on funding mechanisms whether national or international in a context where basic clinical care is scanty at best, and too often unavailable except in larger towns and cities. Many Brazilian clinical researchers are committed to serving low-income patients, well attuned to PAHO protocols, and engaged in collaborative regional health-tracking that uses their professional expertise. Nonetheless, their ability to investigate and serve local and regional patient populations in terms of environmental susceptibility (epigenetic, genetic, and other postulated causal mechanisms, as well) depends in some measure on the availability of funding and research infrastructure that becomes available and is also withdrawn on globalized agendas they do not control. Gibbon's is an important insight that should be part of any qualitative laboratory/clinic-based study: whatever we understand of the local situation is nested within much larger networks of resources and power arrangements to which our scientific collaborators rarely have access.

This is true even in rich laboratories of the global north, as Stephanie Lloyd and Eugene Raikhel demonstrate in their ethnographic account of a Canadian research group studying epigenetics in the brains of "suicide completers." The lab is highly specialized, its work focused through its access to valuable brain tissue: suicide brains are a special and rare resource whose affordances set the limits on what lab members can think, say, and do. These authors provide an extended case study of how epigenetic data are produced and interpreted when searching for brain-based material traces of mechanisms that might shape suicidality.

The virtues of the ethnographic methods are clearly on display in their article: Lloyd and Raikhel hung out long enough to watch postdocs hone their craft, depart to work on other projects, and return to this lab. The result is a highly nuanced and technically proficient investigation of how creativity, collaboration, and competition are all braided through the life cycle of both individual scientific careers and laboratories. The postdoc whose 
observations give title to the article ("It was there all along”) describes finding a difference in an epigenomic sequence in brain tissue of suicide completers that had not previously appeared in the literature. She reasons that the difference may be an artifact of tissue preparation, in which case, a published report might open the lab to charges of sloppy method. Or it may indicate a real divergence, in which case, lab members would have to change their focus to investigate it, should she prevail in reporting it. Either way, her finding risks perturbation in laboratory life, so she sets it aside. Years later, another lab reports virtually the same finding in its publication, leading the former postdoc to speculate on what impact her discovery might have had at the time she found it, if she had pursued its implications across laboratory hierarchy and collaboration. Appropriately, the subtitle of Lloyd and Raikhel's article is "Situated uncertainty and the politics of publication in environmental epigenetics.”

In classic 'grain of mustard seed' fashion, our authors use the former postdoc's modest musings to reveal the larger structure of material constraints and resources of laboratory life: an analysis of everything from local, national, and international funding and tissue monopolies to scientific career-spans can be unpacked from her singular technical dilemma. The charge of inept data production or having your data interrogated when you cannot share it (for reasons specific to this particular brain-holding lab) set the limits on how hunches, doubts, and possible breakthroughs might be expressed. Daily practices of science are described in highly nuanced terms, once the lifespan of scientists, varied lab specialties, and research resources are taken into account. When others report similar findings, the former postdoc regretfully ruminates, "it was there all along." Yet her discovery could not have been expressed in this particular lab at the precise moment when she found it without causing considerable disruption. As our authors point out, the problem of how to contextualize and interpret artifactual findings is widespread in experimental science; but legitimating the search for tissue-specific epigenetic sites, especially in the brain, is particularly fraught amongst researchers deploying epigenetic methodology at the time this particular research takes place. Most importantly, the highly specialized mission and resources of this lab cannot be put into question by the postdoc, thus revealing the seamlessness of externalities to internalities in this (and perhaps most or all) scientific investigation. Thus, the contingent research resources, social practices, hierarchical standings, and lifespans of scientific projects constitute material bedrock on which this project in epigenetics is built.

The seamlessness of external/internal forces shaping laboratory life is also revealed in "The Paradox of Care in Behavioral Epigenetics: Constructing Early-Life Adversity in the Lab.” Martine Lappé focuses on daily practices of care in epigenetic animal research, where researchers test tissue samples of mouse brains, placentas, and gonads in search of the epigenetic traces of experimentally induced stress and maternal/pup separation. Lab manipulations of maternal/pup bonding lead to aggression, depression, and other presumed negative traits across the generations, a finding they believe can plausibly be analogized to humans, measured as Adverse Childhood Experiences (captured on a standardized ACES scale). But in reducing human maternal behavior to lab animal experiments simulating inattention or abandonment, researchers leave out the environmental violence, discrimination, poverty, bad nutrition, and more that clearly affect human (rather than lab rodent) maternal stress, especially in low-income populations. And of course, such models of care 
(or its disruption) focus exclusively on maternal contributions, leaving wider networks of support, including paternal intervention, unacknowledged amongst real-time human communities as they cope with stress. These social relations are absent from experimental design or laboratory discourse (this is a subject that Martine Lappé addresses in other publications).

Lappé shows us that care matters on many levels. The biological effects of care are being investigated as an aspect of knowledge production concerning intergenerational transmission; care here will have an impact on postgenomic knowledge-making more generally. Lappé usefully credits an active lineage of feminist STS philosophers and social science researchers for mobilizing care as a subject of inquiry; her own work makes a contribution to this insistence on widening the processual focus, including her fluent descriptions of gender assumptions that operate in the daily practices of researchers as they handle and manipulate their animal doppelgangers. Nonetheless, this almost-exclusive focus on analogizing dam/pup to human mother/fetal-to-infant care remains scientifically powerful and efficaciously reductive in the best senses of those terms. As Lappé notes, "Care in the lab is simultaneously stabilizing and potentializing, active and passive, restricting and promissory.”

Of course, one could argue that rodent-human analogies of maternal intergenerational transmission of stress/health disorders lie behind the best-developed claims in epigenetic research. This thought style undergirds $\mathrm{DOHaD}$, the theory of "Developmental origins of health and disease." There, debates about what constitutes “ordinary" versus extraordinary stress and how it may be expressed via tissue-specific epigenetic intergenerational non-DNA transmission have long analogized laboratory animal behavioral experiments to human "natural" experiments like the infamous Dutch Hunger Winter. Then, Nazi blockades starved the Dutch population, and children born to women who had been pregnant during that period not only grew to adulthood with significant health deficits, but transmitted those disorders to their own children, despite adequate nutrition and social support in the second generation. This field has a well-developed literature and corresponding debates. Yet some other fields are just beginning to take on epigenetic research programs; they may be more open to the social complexity critiques offered by fellow-traveling social scientists.

At least, that is the more optimistic reading offered in "Epigenetics and Aging Research: Between Adult Malleability and Early Life Programming.” Ruth Müller and Georgia Samaras are studying the field of human aging/gerontology as it increasingly encounters and takes on board epigenetic tools and theories. For our authors, new aging research opens up not only epigenetic shaping of lifespan but also of health span; Epigenetic processes occur at a juncture where basic and pathological processes meet, that is, researchers are tuned in to both normal and accelerated aging. Collectively, various epigenetic gerontology research projects intend to identify biocultural process that retard or accelerate aging; ultimately, they aim to develop interventions into these. In a series of interviews and conferences attended with biogerontology researchers, they find two different kinds of epigenetic thinking at work. In the first, an emergent paradigm of "adult malleability" increasingly expresses the idea that "healthy aging" may retard deterioration through individual lifestyle interventions in nutrition, exercise, social life, and more. There, the goal is to "grab the minute hand of the epigenetic clock and retard its incessant progress" (Gibbs, 2014, p. 170 quoted in Müller and Samaras, this issue). In the second, by contrast, "aging begins before birth,” as it is expressed 
in developmental programming (DOHaD) differentially affecting populations and subpopulations. Like the ACES rodent-human studies on which Martine Lappé reports, this aging research is sensitive to intergenerational transmission. For some investigators, the goal is to tutor low SES high-stress young adults in the ways of healthful living. They themselves may have inherited a "health-span deficit" from the biological stressors transmitted by their immediate ancestors; yet they may nonetheless be able to break the cycle of such heritability for their own progeny if the tutelage of gerontology research pays off. The two strands of aging research operate on what Müller and Samaras identify as "different epistemological and biopolitical scales.” Aging-as-adult-malleability operates on an individual scale; developmental programming operates on populations and sub-populations. The first puts its faith in lifestyle and pharmacological interventions to promote "surplus health" by slowing down aging in individuals; the second seeks to interrupt intergenerational transmission of ill-health for populations with "health deficits." Our authors find an admirable openness to conversation across disciplinary lines. They strongly recommend deep interdisciplinary social science engagement with researchers in biogerontology and clinical old age studies right now, before the stakes in policy surrounding health interventions on aging are solidified. Their recommendation is worth pursuing, full stop. Yet in my own labbased experiences, I've found that many researchers do not have time to become conversant with the health disparities literature, where clear differences in morbidity and mortality are linked to lifelong conditions of discrimination and resource deprivation. Broadly speaking, our collective research on the links of poverty and discrimination to ill-health is more than a century old. Yet many recent syntheses and theoretical discussions of these intractable connections involve analyses of biosocial environments that do not lend themselves to easy measurement as non-reductive variables, a problem for most laboratory-based scientists that all our "special issue" authors have discussed throughout their work.

Stepping back, what lessons might we draw from the collective conversation these articles about "epigenetics at work" open up? As a group, they provide rich empirical analyses and theoretical interpretations that suggest a conundrum: Epigenetics research potentially opens the door to an understanding of intergenerational biosocial entanglements and continual coproductions of life-form variance, including the structures of risk for life-limiting human health impairments. Yet it also frequently shuts that door through implicit conventional cultural assumptions, whether surrounding gender and the complexity of social life; or asserting that quantifiable biological markers and arguments are not only (more easily) measurable but need remain powerfully hegemonic, as well.

In engaging theory and practice in environmental epigenetics, we qualitative researchers confront existential gaps, both old and new. I would argue that there is always an existential gap between the longstanding promissory notes of future perfectibility through scientific research and its translation into medical practice, continually upended by its uneven benefits and dead-ends. This is true not only for social researchers, but also for the literate public, in all its exquisite stratification, as well. Epigenetics holds out the same sorts of therapeutic futurity that gene therapy or oncogenes or antibiotics once held: some of those promises have been powerfully redeemed, sometimes as world-changing interventions. But their biosocial limitations also open up the next round of scientific desire to which various publics are continually being recruited, whether as research subjects, tax-payers, or desperate acolytes at the temple of science. Social analysts of epigenetics and other forms of new 
knowledge production, too, live out this permanently anticipatory scenario. I'd argue that such existential gaps can never be filled; and, indeed, they are becoming increasingly entrenched as the global capitalization of the life sciences moves forward with intensifying velocity, trailing heterogeneous disruptive aspirations in its wake. 'We' readers of BioSocieties know this lesson concerning the structure of existential gaps quite well: It continually mobilizes our social theory conversations, many deemed too abstract or abstruse to pique the engagement of the life scientists in whose labs we are doomed to rediscover these lessons.

A recognition of this permanent gap should not index a self-congratulatory Olympian stance regarding these well-rehearsed existential dramas in the history of scientific research, clinical medicine, and technological innovation. To the contrary, we have no choice but to engage again and again in the hopes that some of the pieces of the infinite jigsaw puzzle of vitality now being viewed through an epigenetic lens may benefit from our biosocial collaborations, critiques, and occasional interventions. Reading these rich essays, we are once again returned to that harrowing recommendation so long ago offered by Gramsci: "Pessimism of the intellect; optimism of the will."

\section{About the Author}

Rayna Rapp is professor of anthropology, New York University, the editor/ author of four books, including Testing Women, Testing the Fetus: the Social Impact of Amniocentesis in American (1999), and is currently co-writing Disability Worlds with Faye Ginsburg. 